\title{
Study on the Design Criteria of the Coaxial Injector for a LOX/Hydrogen Rocket Engine -Evaluation of Design for Injectors of the LE-5 Engine and Its Derivatives-
}

\author{
By Nobuyuki YATSUYANAGi* \\ Formerly, Kakuda Research Center, National Aerospace Laboratory, Kakuda, Japan
}

(Received December 12th, 2006)

\begin{abstract}
Based on the author's recent study on detailed modeling of the atomization characteristics of a liquid/gas phase coaxial injector, an improved computation model for predicting the combustion performance of a LOX/hydrogen engine is proposed. The features of this model are that it allows calculation of the local rate of atomization of the LOX jet and deals with droplet size distribution of the LOX spray. Additionally the model applies the burning rate constant of LOX/hydrogen combustion derived by the author's former experiment. Using this model, evaluations of the design criteria for the LE-5 engine, which was equipped on the H-2 launcher, and its derivative engines, which have been used with the H-2A launcher, were conducted. Furthermore, optimization of the derivative engine's injector design to improve combustion stability and combustion performance was discussed.
\end{abstract}

Key Words: $\quad$ LOX/Hydrogen Engine, Coaxial Injector, $C^{*}$ efficiency, Combustion Stability

\section{Nomenclature}

$A_{i}$ : cross-sectional area of any feed line

$A_{\mathrm{t}}$ : nozzle throat area

$A(x)$ : cross-sectional area of gas phase

$\bar{a}(x)$ : mean acceleration of droplet

$C^{*}$ : characteristic exhaust velocity

$\begin{aligned} D(x): & \text { droplet diameter } \\ E_{\mathrm{cr}}: & \text { critical Eötvös number }\left(=\frac{\bar{a}(x) \rho_{1} D^{2}(x)}{g \sigma_{1}}=1.3\right) \\ g: & \text { gravity acceleration }\end{aligned}$

$g$ : gravity acceleration

$j$ : imaginary unit

$l_{i}$ : length of any feed line

$M$ : molecular weight of combustion gas

$M_{\mathrm{c}}$ : Mach number

$N_{\mathrm{u}}(x)$ : Nusselt number

$O / F$ : mixture ratio

$R(x)$ : outer boundary of LOX core

$\Re$ : universal gas constant

$s$ : Laplace operator

$T_{\mathrm{c}}$ : combustion gas temperature

$t$ : time

$U(x)$ : velocity

$V_{i}$ : volume of any feed line

$V_{\mathrm{c}}$ : volume of combustion chamber

$\rho$ : density

$\gamma_{\mathrm{c}}$ : specific heat ratio of combustion gas

$\sigma_{1}$ : surface tension of LOX

$\omega$ : angular frequency

$\eta_{C^{*}}: C^{*}$ efficiency

$\Delta:$ increment or disturbance

(C) 2007 The Japan Society for Aeronautical and Space Sciences

${ }^{*}$ Present address, 989-1603, Miyagi, Japan

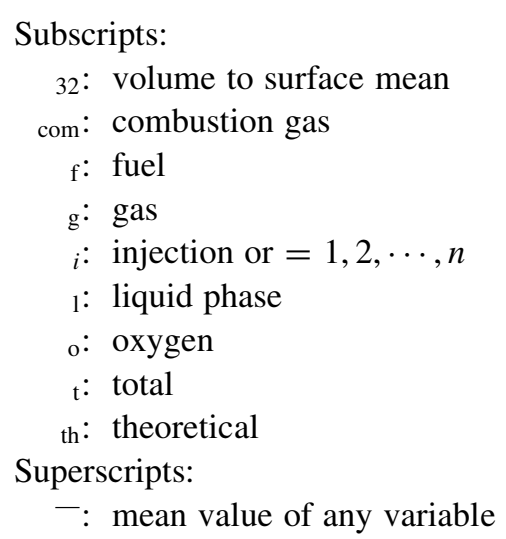

\section{Introduction}

Japan developed the H-2 and H-2A rockets in the 1980s and 1990s, respectively. In the development of these rockets, the key developing item was LOX/hydrogen engines with a higher specific impulse, $I_{\mathrm{SP}}$. Thus, an LE-5 engine with an $I_{\mathrm{SP}}$ of $440 \mathrm{sec}$ was developed for use as the upperstage engine of the H-2 rocket, and an LE-7 engine having an $I_{\mathrm{SP}}$ of $445 \mathrm{sec}$ was developed for use as the booster engine for the H-2A rocket. Furthermore, the LE-5 derivative engines were developed as alternate engines to improve the launching performance of the $\mathrm{H}-2 \mathrm{~A}$ rocket. With the development of these LOX/hydrogen engines, evaluation of the LOX/hydrogen rocket engine design, especially that of the coaxial injector and combustion chamber, has become important for understanding the design criteria necessary for further development, including that of a future LOX/ LNG engine. In the author's former study, ${ }^{1)}$ an analytical computation model to calculate the combustion performance of the LOX/hydrogen engine with a coaxial injector 
was derived. In that model, the process of the LOX jet's atomization, that of the mixing of the LOX spray with the gaseous hydrogen, and that of the vaporization of the LOX droplets were considered as the controlling factors affecting combustion performance. However, the assumed modeling of the atomization process of the LOX jet was simplified as the constant rate of atomization for the region of atomization, because reliable modeling of that process had not yet been studied at the time. In other typical models of combustion, ${ }^{2,3)}$ instantaneous atomization at the exit of the injector elements and the mono-dispersed size of the LOX spray were considered, and the quasi-steady state vaporization of the representative droplet in a forced convection environment was applied to calculate the amount of reacted mass of propellant. In the author's recent study, ${ }^{4}$ however, a detailed model of the atomization characteristics of the coaxial injector of the liquid/gas phase, including the local rate of atomization and the size distribution of formed spray, was developed. Thus, in this study, an improved calculation model of combustion performance is developed by adopting this detailed mechanism of atomization ${ }^{4}$ and the burning rate constant obtained in the author's former experiment. ${ }^{5)}$ Using this proposed model for the combustion process, not only the evaluation of combustion performance, but also that of combustion stability is possible. In the present paper, the improved combustion process model is described, and then the combustion performance and combustion stability of the LE-5 engine and its derivative engines are evaluated and discussed.

\section{Analytical Model of Combustion Characteristics of a LOX/Hydrogen Engine with a Coaxial Injector}

\subsection{Analytical model of combustion performance}

First, an outline of the author's former model for predicting the combustion performance of a LOX/hydrogen engine with a coaxial injector ${ }^{1)}$ is presented. A simplified illustration of the assumed model of the combustion scheme is presented in Fig. 1, which shows that the combustion zone is divided into a series of two sub-zones along the axis of the combustion chamber. In the region immediately adjacent to the injector face (i.e., region-1), the flow was characterized by the behavior of the LOX spray jet from the injector elements. After each spray jet interacted with each other

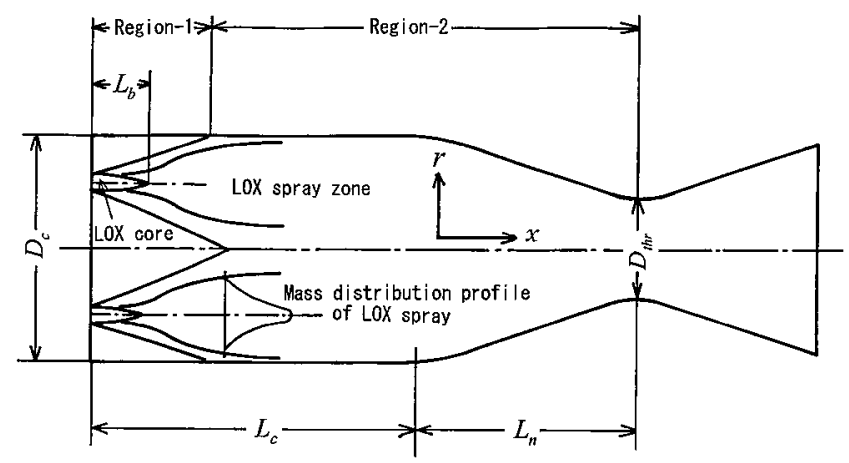

Fig. 1. Simplified illustration of assumed model of combustion scheme. (i.e., region-2), the flow was assumed to be almost onedimensional except for the mass flux distribution of the LOX spray in space. For region-1, a constant rate of atomization was assumed till completion of the LOX jet's breakup, where the breakup length of the LOX jet was based on the author's previous experiment. ${ }^{6}$ In region-2, the process of the steady-state vaporization of the LOX droplets of a mono-sized mean diameter and the process of the mixing between vaporized oxygen with the injected hydrogen flow were considered as controlling factors affecting combustion performance. By that analysis, the predicted characteristic exhaust velocity efficiencies, $\eta_{C^{*}}$, were almost within $\pm 2 \%$ for the LE-5, RL-10 (used for the Centaur rocket of the US) and HM-7 (used for the Ariane rocket of Europe) engines. In the present study, on the other hand, an improved model of the atomization process, ${ }^{4)}$ which enables the calculation of the local atomization rate of LOX jet, breakup length of it, $L_{\mathrm{b}}$, histogram of the droplet's size distribution and mean diameter of the LOX spray, $D_{32}$, is applied to improve the predicting model of combustion performance. An outline of the present model for atomizing a LOX jet with hydrogen gas flow is as follows. In this model, the local rate of atomization from the core of the LOX jet and the size of the droplets of the LOX spray are calculated based on onedimensional conservation of momentum at the interaction surface between the LOX jet and gas hydrogen flow, as well on the correlation of the critical Eötvös number, ${ }^{7)}$ which shows the correlation between the equilibrium size of the droplets and the acceleration of droplets, and this is represented in the nomenclature. As a result, the thickness of the stripping layer from the core of the LOX jet, $\Delta R(x)$, which is eventually atomized and subsequently forms the spray for the increment of axial distance, $\Delta x$, is given as the solution of Eq. (1). The local rate of atomization of the LOX jet at arbitrary $x$ is given by Eq. (2). The initial diameter of the droplets formed at $x$ is given by Eq. (3), and the number of droplets is given by Eq. (4), where $c_{\mathrm{a}}$ and $c_{\mathrm{m}}$ are empirical constants (i.e., $c_{\mathrm{a}}=0.02$ and $c_{\mathrm{m}}=500$ ).

$$
\begin{aligned}
& \frac{\Delta R(x)}{R(x)}=\frac{c_{\mathrm{m}} \rho_{\mathrm{g}} \bar{U}_{\mathrm{g}}^{2}(x) \Delta A(x)}{\rho_{\mathrm{l}} U_{\mathrm{l}}^{2}(x) A(x)}-\frac{c_{\mathrm{a}}{ }^{-1} \sigma_{\mathrm{l}} g E_{\mathrm{cr}}}{\rho_{\mathrm{l}} U_{\mathrm{l}}^{2}(x) \Delta R(x)} \\
& \Delta W_{\mathrm{a}}(x)=2 \pi R(x) \Delta R(x) \Delta x \rho_{\mathrm{l}} \\
& D^{2}(x)=c_{\mathrm{a}} \Delta R(x) \Delta x \\
& N_{\mathrm{d}}(x)=\Delta W_{\mathrm{a}}(x) / \frac{\pi}{6} D^{3}(x) \rho_{\mathrm{l}}
\end{aligned}
$$

In this way, the proposed atomization model can deal with the LOX spray having a various sizes of droplets, rather than as a mono-sized spray as adopted in the former study. Additionally, in this combustion model, the burning rate constant that was derived in the author's former experiment is applied to calculate the amount of reacted mass of the propellants in region-2. In that experiment, ${ }^{5)}$ the burning rate constants of the LOX spray with gaseous hydrogen, $K_{\mathrm{b}}$ $\left(\mathrm{m}^{2} / \mathrm{s}\right)$, were obtained, where $K_{\mathrm{b}}$ was deduced from the correlation of the squares of representative LOX diameter vs. the corresponding burning time. This is based on the fact 
Table 1. Dimensions of injectors and combustors, and operating conditions.

\begin{tabular}{|c|c|c|c|c|c|}
\hline & (unit) & CASE-A & CASE-B & CASE-C $\#$ & CASE-D $^{\#}$ \\
\hline Number of injection elements & - & 208 & 127 & 208 & 127 \\
\hline Dimension of injection & $d_{\mathrm{o}}(\mathrm{mm})$ & 2.5 & 3.3 & 2.8 & 5.3 \\
\hline \multirow[t]{3}{*}{ element } & $d_{n}(\mathrm{~mm})$ & 3.5 & 4.3 & 3.5 & 6.3 \\
\hline & $d_{\mathrm{f}}(\mathrm{mm})$ & 4.6 & 5.9 & 4.6 & 6.9 \\
\hline & $L_{\mathrm{rec}}(\mathrm{mm})$ & 2.5 & 3.3 & 2.5 & 5.3 \\
\hline Chamber dia. & $D_{\mathrm{c}}(\mathrm{m})$ & 0.24 & $\rightarrow$ & $\rightarrow$ & $\rightarrow$ \\
\hline Throat dia. & $D_{\text {thr }}(\mathrm{m})$ & 0.136 & $\rightarrow$ & 0.142 & 0.160 \\
\hline \multirow[t]{2}{*}{ Chamber length } & $L_{\mathrm{c}}(\mathrm{m})$ & 0.107 & $\rightarrow$ & 0.235 & 0.286 \\
\hline & $L_{n}(\mathrm{~m})$ & 0.202 & $\rightarrow$ & 0.101 & 0.176 \\
\hline Combustion pressure & $P_{\mathrm{c}}(\mathrm{MPa})$ & 3.50 & $\rightarrow$ & 3.97 & 3.57 \\
\hline \multirow[t]{2}{*}{ Injection pressure } & $P_{\mathrm{o}, i}(\mathrm{MPa})$ & 4.67 & $\rightarrow$ & 5.46 & 4.78 \\
\hline & $P_{\mathrm{f}, i}(\mathrm{MPa})$ & 4.02 & $\rightarrow$ & 4.75 & 4.79 \\
\hline \multirow[t]{2}{*}{ Interface pressure } & $P_{\mathrm{o}, \mathrm{t}}(\mathrm{MPa})$ & 5.01 & $\rightarrow$ & 5.73 & 5.14 \\
\hline & $P_{\mathrm{f}, \mathrm{t}}(\mathrm{MPa})$ & 4.07 & $\rightarrow$ & 4.79 & 4.84 \\
\hline LOX flow rate & $W_{\mathrm{o}, i}(\mathrm{~kg} / \mathrm{s})$ & 18.9 & $\rightarrow$ & 22.7 & 26.0 \\
\hline Hydrogen flow rate & $W_{\mathrm{f}, i}(\mathrm{~kg} / \mathrm{s})$ & 3.1 & $\rightarrow$ & 4.1 & 4.5 \\
\hline \multirow[t]{2}{*}{ Injection velocity } & $U_{\mathrm{o}, i}(\mathrm{~m} / \mathrm{s})$ & 17.1 & 15.3 & 15.8 & 8.2 \\
\hline & $U_{\mathrm{f}, i}(\mathrm{~m} / \mathrm{s})$ & 343 & 305 & 379 & 322 \\
\hline \multirow[t]{2}{*}{ Injection temperature } & $T_{\mathrm{o}, i}(\mathrm{~K})$ & 94 & $\rightarrow$ & $\rightarrow$ & $\rightarrow$ \\
\hline & $T_{\mathrm{f}, i}(\mathrm{~K})$ & 120 & $\rightarrow$ & 131 & 57 \\
\hline
\end{tabular}

\#Values of some of the data are presumed.

that the square law of the droplet vs. burning time was examined for the LOX spray of a rocket's combustion. Thus, in a forced convection environment, the relation of $D(x)^{2}$ to $K_{\mathrm{b}}$ is given by the following:

$$
\Delta D(x)^{2} / \Delta t=-K_{\mathrm{b}} N_{\mathrm{u}}(x) / 2
$$

In the present paper, therefore, $K_{\mathrm{b}}=1.1 \times 10^{-5}\left(\mathrm{~m}^{2} / \mathrm{s}\right)$ is applied to calculate the amount of reacted mass from each droplet of LOX spray, which corresponds to a combustion pressure of 3.5 MPa at a Nusselt number of 2. The amount of propellant which reacted within the increment of $\Delta x$ at an arbitrary $x$ is given by summing up the reacted masses of all droplets forming the spray. The other assumptions made in the present study are the same as those of the author's former combustion model. ${ }^{1)}$

\subsection{Analytical model of combustion stability}

In a series of development firing tests of the LE-5 and its derivative engines, combustion in all of the engines except one derivative engine with a candidate injector, CASE-D in Table 1, were stable. The engine of CASE-D showed rather unstable combustion with a fluctuation in combustion pressure of $15 \%$ peak-to-peak in amplitude to a steady state. Vibration of the engine at a frequency of around 40$60 \mathrm{~Hz}$ was also observed. Therefore, in the present study, an evaluation of combustion stability is conducted for the injectors of the LE-5 and its derivative engines. Here, the analytical model derived by Szuch $^{8)}$ is applied to focus on low-frequency combustion instability. This model is based on the fact that low-frequency combustion instability is caused by a coupling of the propellant feed system with the combustion dynamics in such a way as to reinforce any disturbance in pressure or propellant flow. In a system having time delays associated with the combustion processes of bipropellants, a characteristic equation which correlates a small disturbance in the rate of change of burned products, $\Delta w_{\mathrm{b}}$, to the disturbance in combustion pressure, $\Delta P_{\mathrm{c}}$, had been derived. Thus, without the time delays, combustion is essentially stable. Therefore, the combustion time delays become the most critical factor for determining combustion stability. In Szuch's model, ${ }^{8)}$ the combustion time delays were evaluated by Priem-Heidmann's model ${ }^{2)}$ of combustion, which was based on the vaporization process of the LOX spray in mono-dispersed size. In the present study, however, the combustion time delays calculated by this author's combustion model are applied to elucidate the details of the time delays. Namely, the combustion time delay for the LOX spray, $\tau_{\mathrm{o}}$, is mainly based on the time necessary for atomization of the LOX jet, $\tau_{\mathrm{a}}$, and the time needed for completion of LOX droplet combustion, $\tau_{\text {com }}$, which includes the time necessary for completion of LOX droplet vaporization and for mixing these with the injected gaseous hydrogen. This is defined by Eq. (6). For gaseous hydrogen, only the mixing delay time, $\tau_{\text {mix }}$, is responsible for combustion time delay, Eq. (7), and this is on the order of $0.1 \mathrm{~ms}$ by Szuch's model ${ }^{8)}$ Here, $\tau_{\text {mix }}=0.1 \mathrm{~ms}$ is assumed for all cases.

$$
\begin{aligned}
& \tau_{\mathrm{o}}=\tau_{\mathrm{a}}+\tau_{\mathrm{com}} \\
& \tau_{\mathrm{f}}=\tau_{\mathrm{mix}}
\end{aligned}
$$

In Fig. 2, a schematic diagram of the analysis by Szuch is shown. Here, the transfer function, which correlates a small disturbance of burning rate, $\Delta w_{\mathrm{b}}(s)$, with the growth of the 


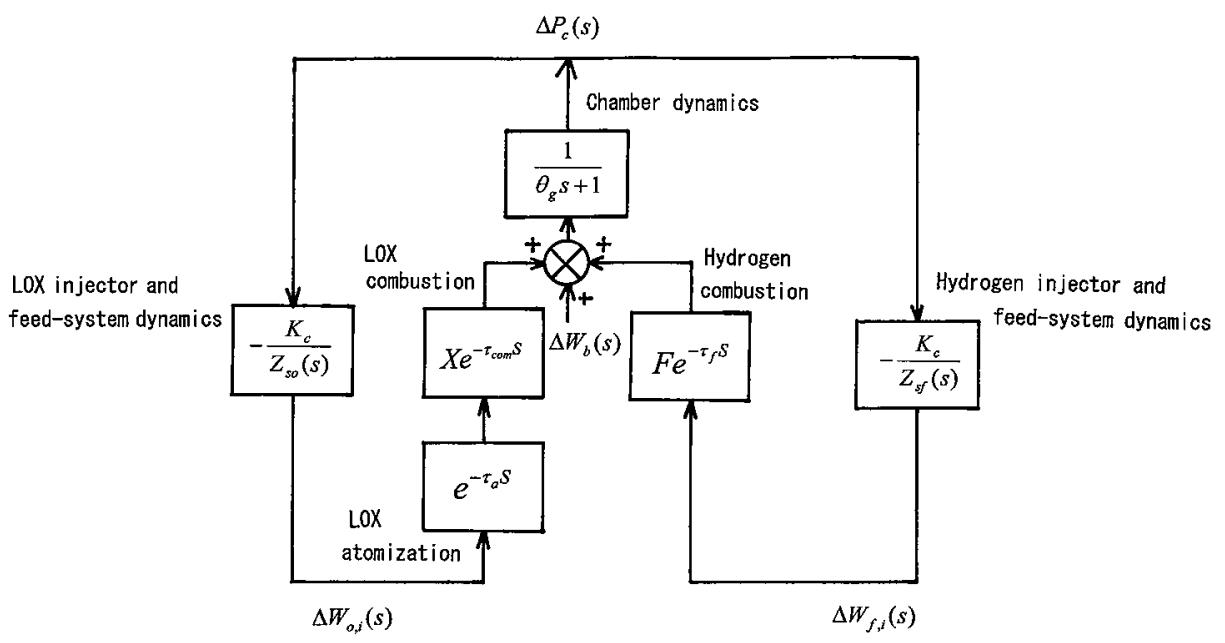

Fig. 2. Schematic diagram of an analytical model of combustion instability by Szuch. ${ }^{8}$

amplitude of combustion pressure oscillation, $\Delta P_{\mathrm{c}}(s)$, is expressed by Eq. (8). The gain of the transfer function, $|G(j \omega)|$, is expressed by Eq. (9), and the difference of the phase angle, $\Theta$ (i.e., the phase margin) between $\Delta P_{\mathrm{c}}(s)$ and $\Delta w_{\mathrm{b}}(s)$, is expressed by Eq. (10). Additionally, $\theta_{\mathrm{g}}$ is the combustion gas residence time in the combustion chamber ${ }^{9)}$ and is given by Eq. (11). The complex flow impedances, $Z_{\mathrm{so}}(s)$ and $Z_{\mathrm{sf}}(s)$, are presented by Eq. (12) and Eq. (13), respectively, if the geometrical dimensions for the LOX feed lines and those of the hydrogen gas lines are given.

$$
\begin{aligned}
& \frac{\Delta P_{\mathrm{c}}(s) / P_{\mathrm{c}}}{\Delta w_{\mathrm{b}}(s) / w_{\mathrm{t}}} \\
& \quad=\frac{1}{\theta_{\mathrm{g}} s+1+\left(X K_{\mathrm{c}} / Z_{\mathrm{so}}(s)\right) e^{-\tau_{\mathrm{o}} S}+\left(F K_{\mathrm{c}} / Z_{\mathrm{sf}}(s)\right) e^{-\tau_{\mathrm{f}} S}}
\end{aligned}
$$

$|G(j \omega)|$

$$
=\frac{1}{\sqrt{\left(1+A_{\mathrm{o}} Y_{\mathrm{Ro}}+A_{\mathrm{f}} Y_{\mathrm{Rf}}\right)^{2}+\left(\theta_{\mathrm{g}} \omega+A_{\mathrm{o}} Y_{\mathrm{Io}}+A_{\mathrm{f}} Y_{\mathrm{If}}\right)^{2}}}
$$$$
\Theta=\tan ^{-1}\left(-\frac{\theta_{\mathrm{g}} \omega+A_{\mathrm{o}} \times Y_{\mathrm{Io}}+A_{\mathrm{f}} \times Y_{\mathrm{If}}}{1+A_{\mathrm{o}} \times Y_{\mathrm{Ro}}+A_{\mathrm{f}} \times Y_{\mathrm{Rf}}}\right)
$$

$$
\theta_{\mathrm{g}}=M V_{\mathrm{c}} C^{*} /\left(\Re T_{\mathrm{c}} A_{\mathrm{t}}\right)
$$

where,

$$
\begin{aligned}
& A_{\mathrm{o}}=X K_{\mathrm{c}} /\left(R_{\mathrm{o}}{ }^{2}+I_{\mathrm{o}}{ }^{2}\right), \quad A_{\mathrm{f}}=F K_{\mathrm{c}} /\left(R_{\mathrm{f}}{ }^{2}+I_{\mathrm{f}}{ }^{2}\right) \\
& X=\eta_{C^{*}} / A_{\mathrm{t}} g\left\{C_{\mathrm{th}}{ }^{*}+[(O / F)+1] \partial C_{\mathrm{th}}{ }^{*} / \partial(O / F)\right\} \\
& F=\eta_{C^{*}} / A_{\mathrm{t}} g\left\{C_{\mathrm{th}}{ }^{*}-(O / F)[(O / F)+1] \partial C_{\mathrm{th}}{ }^{*} / \partial(O / F)\right\} \\
& K_{\mathrm{c}}=\frac{1+\gamma_{\mathrm{c}} M_{\mathrm{c}}{ }^{2}}{\left[1+\left(\gamma_{\mathrm{c}}-1\right) M_{\mathrm{c}}{ }^{2} / 2\right]^{\gamma_{\mathrm{c}} /\left(\gamma_{\mathrm{c}}-1\right)}}
\end{aligned}
$$

and,

$$
\begin{aligned}
& Y_{\text {Ro }}=R_{\mathrm{o}} \times \cos \left(\tau_{\mathrm{o}} \omega\right)-I_{\mathrm{o}} \times \sin \left(\tau_{\mathrm{o}} \omega\right) \\
& Y_{\mathrm{Io}}=-R_{\mathrm{o}} \times \sin \left(\tau_{\mathrm{o}} \omega\right)-I_{\mathrm{o}} \times \cos \left(\tau_{\mathrm{o}} \omega\right) \\
& Y_{\mathrm{Rf}}=R_{\mathrm{f}} \times \cos \left(\tau_{\mathrm{f}} \omega\right)-I_{\mathrm{f}} \times \sin \left(\tau_{\mathrm{f}} \omega\right) \\
& Y_{\mathrm{If}}=-R_{\mathrm{f}} \times \sin \left(\tau_{\mathrm{f}} \omega\right)-I_{\mathrm{f}} \times \cos \left(\tau_{\mathrm{f}} \omega\right)
\end{aligned}
$$



Fig. 3. Schematic of injector element configuration.

$$
\begin{aligned}
& Z_{\mathrm{so}}(j \omega)=R_{\mathrm{o}}+j I_{\mathrm{o}} \\
& Z_{\mathrm{sf}}(j \omega)=R_{\mathrm{f}}+j I_{\mathrm{f}}
\end{aligned}
$$

Combustion stability is then assured if the gain of the transfer function is negative with a proper phase margin in the frequency range of interest. In general, the existence of combustion time delays may trigger unstable combustion, however, if once combustion instability occurs, then the process of the atomization and combustion are greatly affected. So the discussion by the analytical model should be understood as a qualitative one.

\section{Evaluation of Design for the Injectors of the LE-5 and Its Derivative Engines}

\subsection{Evaluation of combustion performance}

Using the present model, the $C^{*}$ efficiency, which represents the combustion efficiency, is calculated for the LE-5 and its derivative engines to evaluate the design criteria of the injector. The configuration and dimensions of the injector element and the operation conditions are shown in Fig. 3 and Table 1, respectively. CASE-A is that of the LE-5 engine, CASE-B is that of the LE-5 engine with a candidate injector, CASE-C and CASE-D are those of the LE-5 derivative engines with a candidate injector. Some values of these data listed in Table 1 are presumed based on a reference, ${ }^{4)}$ because the details have not been published. Typical calculated results are shown in Figs. 4-6 and Table 2. 


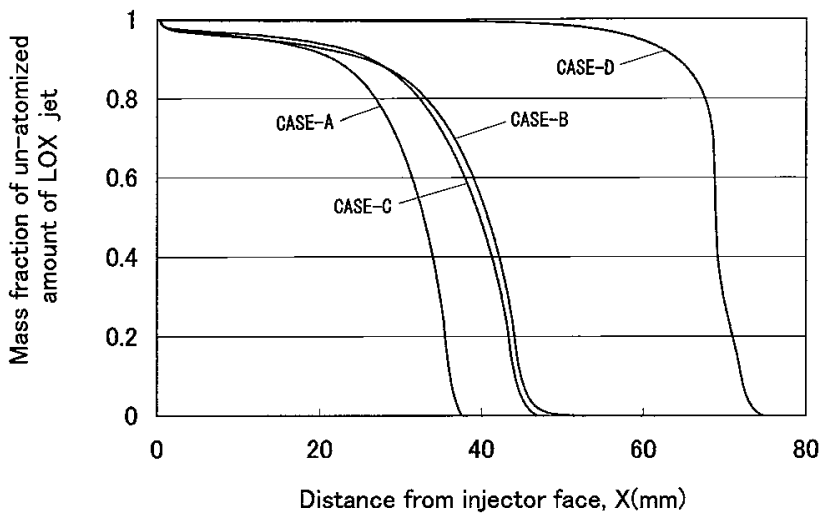

Fig. 4. Calculated mass fraction of un-atomized amount of LOX jet.



Fig. 5. Calculated histogram of LOX droplet's size distribution at $x=L_{\mathrm{b}}$.

Figure 4 shows the calculated results for the mass fraction of the un-atomized amount of LOX jet $(=1-$ $\left.\sum_{x=0}^{L_{\mathrm{b}}} \Delta W_{\mathrm{a}}(x) / W_{\mathrm{o}, i}\right)$ vs. the distance from the injector face,
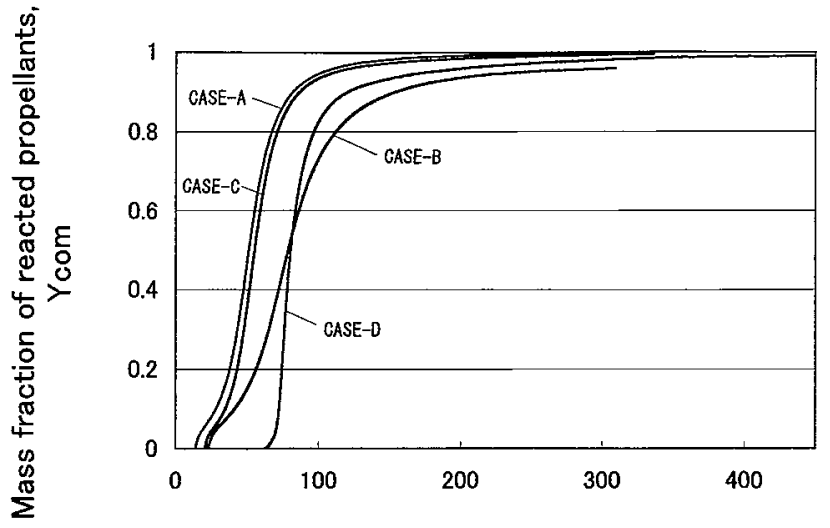

Distance from injector face, $x(\mathrm{~mm})$

Fig. 6. Calculated mass fraction of reacted propellants vs. distance from injector face.

where the length corresponding to the ordinate $=0$ is the breakup length of the LOX jet. It is clear that CASE-D has the longest breakup length. Figure 5 shows the calculated histogram of the LOX droplet's size distribution at $x=L_{\mathrm{b}}$ for each injector. For these LOX spray characteristics, the calculated mass fraction of the reacted propellants, $Y_{\text {com }}$, vs. the distance from the injector face is shown in Fig. 6. In this figure, the profiles of $Y_{\text {com }}$ in CASE-A and CASE-C are almost the same, since the dimensions of the injector elements and the injection conditions are almost the same, so the histograms of the LOX droplet's size distribution are almost same as shown in Fig. 5. In CASE-D, on the other hand, the size of the LOX injection orifice is larger than those of the others and the LOX injection velocity is fairly low, so the atomization length of the LOX jet becomes longer and the formed spray has a much coarser $D_{32}$ than in

Table 2. Calculated results.



${ }^{\#}$ Evaluated at $x=L_{\mathrm{b}}$.

\#\# Performance of prototype engine.

\#\#\# Not published. 




Fig. 7. Configuration of the injector.

the other cases. As a result, the combustion zone becomes far from the injector face. However, the combustion chamber length is longest $\left(L_{\mathrm{c}}+L_{n}=0.46 \mathrm{~m}\right)$ among these cases, so achieved efficiency is better than CASE-A and CASE-B. In principle, it can be said that the size of the LOX injection orifice is the most effective for the atomization and combustion processes under the condition of constant injection mass flow rate of LOX, so for the time to complete combustion, $\tau_{\mathrm{o}}$. In Table 2, the $C^{*}$ efficiencies and atomization time delay of the LOX jet, $\tau_{\mathrm{a}}$, and the combustion time delay, $\tau_{\mathrm{com}}$, are shown, where $\tau_{\mathrm{a}}$ and $\tau_{\mathrm{com}}$ are used as the characteristic times for the analysis of Szuch's combustion stability. As for the $C^{*}$ efficiencies in CASE-A and CASE-B, the calculated values are fairly consistent with the experimental ones, while in CASE-C and CASE-D, the experimental data have not been published. Here the LOX atomization time delay, $\tau_{\mathrm{a}}$, is calculated by $L_{\mathrm{a}} / U_{\mathrm{o}, i}$, and $L_{\mathrm{a}}$ is defined as the length necessary to generate the drops with the Sauter mean diameter, $D_{32}$, of the LOX spray from the injector face. The combustion time delay, $\tau_{\text {com }}$, is defined based on the time necessary to complete the combustion of droplets with a size of $D_{32}$. This is because the Sauter mean diameter is the most suitable to express as the representative diameter for the phenomena of spray combustion. Hereafter, the calculated time delays using the present model, the time needed for atomization of the LOX jet, $\tau_{\mathrm{a}}$, and the time until completion of combustion, $\tau_{\text {com }}$, are used to evaluate combustion stability.

\subsection{Evaluation of combustion stability}

To calculate the gain and phase margin of the characteristic equations, Eqs. (9) and (10), it is necessary to know the impedances of each injector. Regarding the configuration of the injector shown in Fig. 7, the impedance of the LOX feed line and that of the gaseous hydrogen feed line



(a) Impedance representative of LOX feed line.

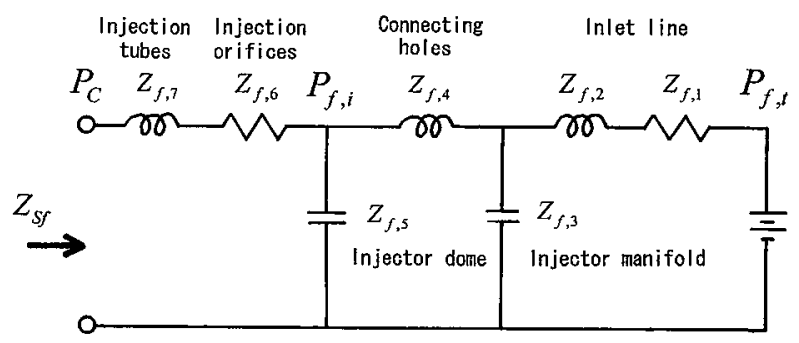

(b) Impedance representative of hydrogen feed line.

Fig. 8. Schematics of impedance representation of feed systems.

are represented in Fig. 8. For each injector, resistances, inductances and capacitances are calculated by the equations listed in Table 3, substituting the concrete numerical values to each equation. Substituting these values into Eqs. (12) and (13), then Eqs. (9) and (10) give the Gain and phase margin of the transfer function, respectively, for an interested range of frequency, $f(\mathrm{~Hz})$, using the calculated $\tau_{\mathrm{o}}$ and $\tau_{\mathrm{f}}$ tabulated in Table 2. The calculated results are shown in Figs. 9 and 10. As shown in these figures, CASE-A,

Table 3. Table of equations to compute complex flow impedance.

\begin{tabular}{|c|c|c|}
\hline \multicolumn{3}{|c|}{ Equations of complex flow impedance*: } \\
\hline$Z_{\mathrm{o}, 1}=2\left(P_{\mathrm{o}, \mathrm{t}}-P_{\mathrm{o}, i}\right) / W_{\mathrm{o}, i}$, & $Z_{\mathrm{o}, 2}=j \omega\left(l_{0,2} / A_{\mathrm{o}, 2} g\right)$, & $Z_{\mathrm{o}, 3}=\rho_{1} V_{\mathrm{o}, 3} / B^{\#}$ \\
\hline$Z_{\mathrm{o}, 4}=2\left(P_{\mathrm{o}, i}-P_{\mathrm{c}}\right) / W_{\mathrm{o}, i}$ & $Z_{\mathrm{o}, 5}=j \omega\left(l_{0,5} / A_{\mathrm{o}, 5} g\right)$ & $Z_{\mathrm{o}, 6}=j \omega\left(l_{0,6} / A_{\mathrm{o}, 6} g\right)$ \\
\hline$Z_{\mathrm{f}, 1}=2\left(P_{\mathrm{f}, \mathrm{t}}-P_{\mathrm{f}, i}\right) / W_{\mathrm{f}, i}$ & $Z_{\mathrm{f}, 2}=j \omega\left(l_{\mathrm{f}, 2} / A_{\mathrm{f}, 2} g\right)$ & $Z_{\mathrm{f}, 3}=\rho_{\mathrm{f}} V_{\mathrm{f}, 3} /\left(\gamma_{\mathrm{f}} P_{\mathrm{f}, i}\right)$ \\
\hline$Z_{\mathrm{f}, 4}=j \omega\left(l_{\mathrm{f}, 4} / A_{\mathrm{f}, 4} g\right)$ & $Z_{\mathrm{f}, 5}=\rho_{\mathrm{f}} V_{\mathrm{f}, 5} /\left(\gamma_{\mathrm{f}} P_{\mathrm{f}, i}\right)$ & $Z_{\mathrm{f}, 6}=2\left(P_{\mathrm{f}, i}-P_{\mathrm{c}}\right) / W_{\mathrm{f}, i}$, \\
\hline$Z_{\mathrm{f}, 7}=j \omega\left(l_{\mathrm{f}, 7} / A_{\mathrm{f}, 7} g\right)$ & & \\
\hline
\end{tabular}

${ }^{*}$ Here, the unit of pressure is $\left(\mathrm{kg} / \mathrm{m}^{2}\right)$.

\#Bulk modulus of LOX, 0.005 is assumed. 




Fig. 9. Gain of transfer function for each injector.

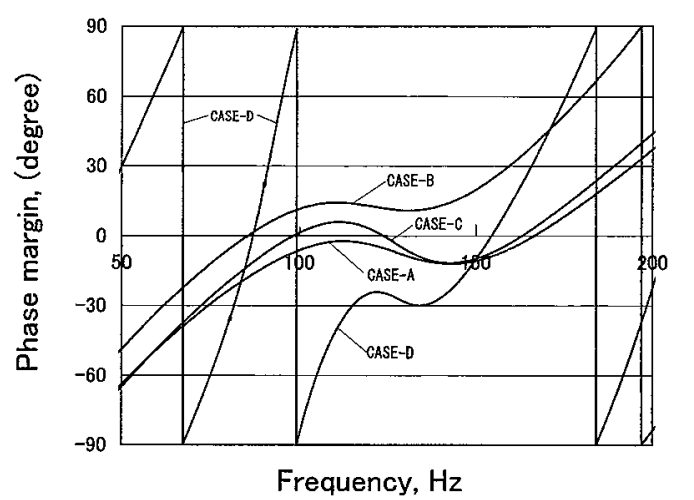

Fig. 10. Phase margin of transfer function for each injector.

Frequency, $\mathrm{Hz}$

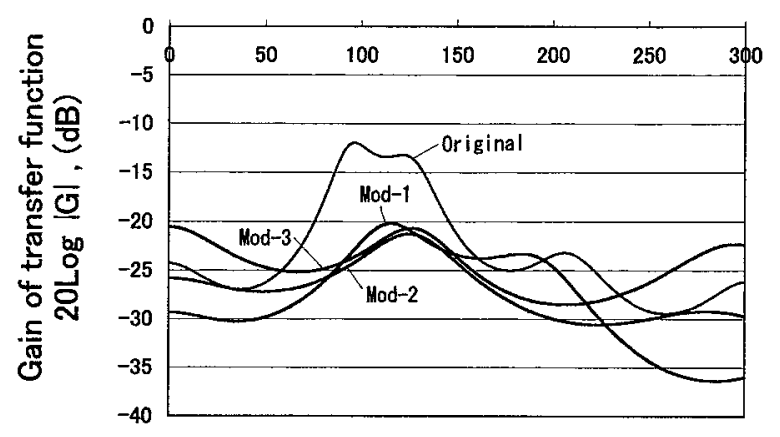

Fig. 11. Optimization of design of injector element to improve combustion stability.

CASE-B and CASE-C show stable combustion, and in fact, stable combustion in these cases was confirmed by firing tests. In CASE-D, however, the predominant peaks of the Gain, although being negative, appear at frequencies of approximately 80 to $120 \mathrm{~Hz}$ in Fig. 9. In Fig. 10, the phase margin passes through zero at nearly 80 and $100 \mathrm{~Hz}$, so this case may be said to be in a marginally stable combustion in the frequency range of around 80 to $100 \mathrm{~Hz}$. Actually, by firing tests, a $15 \%$ peak-to-peak fluctuation in the amplitude of the combustion pressure was observed. Additionally, engine vibration was observed at a frequency of around $40-60 \mathrm{~Hz}$, however, data on the frequency components of combustion pressure fluctuation itself was not published.
Table 4. Improvement in combustion stability of LE-5 derivative engine.

\begin{tabular}{lllll}
\hline & $\begin{array}{c}\text { Original } \\
\left(\text { CASE- }{ }^{\#}\right)\end{array}$ & Mod-1 & Mod-2 & Mod-3 \\
\hline $\begin{array}{l}\text { Number of injection } \\
\text { elements }\end{array}$ & 127 & $\rightarrow$ & $\rightarrow$ & $\rightarrow$ \\
\hline$d_{\mathrm{o}}(\mathrm{mm})$ & 5.3 & 4.5 & 4.0 & 3.5 \\
$d_{n}(\mathrm{~mm})$ & 6.3 & 5.5 & 5.0 & 4.5 \\
$d_{\mathrm{f}}(\mathrm{mm})$ & 6.9 & 6.65 & 6.55 & 5.84 \\
\hline$U_{\mathrm{o}, i}(\mathrm{~m} / \mathrm{s})$ & 8.2 & 11.3 & 14.4 & 18.8 \\
$U_{\mathrm{f}, i}(\mathrm{~m} / \mathrm{s})$ & 322 & 322 & $\rightarrow$ & $\rightarrow$ \\
\hline$T_{\mathrm{f}, i}(\mathrm{~K})$ & 57 & 120 & $\rightarrow$ & $\rightarrow$ \\
\hline$L_{\mathrm{b}}(\mathrm{mm})$ & 74.8 & 53.9 & 47.4 & 40.1 \\
\hline$L_{\mathrm{a}}(\mathrm{mm})$ & 61.5 & 37.7 & 16.8 & 12.0 \\
\hline$D_{32}(\mu \mathrm{m})^{\# \#}$ & 240 & 238 & 240 & 238 \\
\hline$\tau_{\mathrm{a}}(\mathrm{msec})$ & 7.50 & 3.31 & 1.16 & 0.64 \\
\hline$\tau_{\mathrm{com}}(\mathrm{msec})$ & 2.85 & 2.70 & 2.64 & 3.15 \\
\hline$\tau_{\mathrm{o}}(\mathrm{msec})$ & 10.35 & 6.01 & 3.80 & 3.79 \\
\hline$\tau_{\mathrm{f}}(\mathrm{msec})$ & 0.1 & $\rightarrow$ & $\rightarrow$ & $\rightarrow$ \\
\hline$\theta_{\mathrm{g}}(\mathrm{msec})$ & 1.47 & $\rightarrow$ & $\rightarrow$ & $\rightarrow$ \\
\hline$\eta_{C^{*}}\left(=C_{\mathrm{cal}}{ }^{*} / C_{\mathrm{th}}{ }^{*}\right)$ & 0.991 & 0.996 & 0.997 & 0.994 \\
\hline$V_{\mathrm{n}}$ & & &
\end{tabular}

\#Values of some of the data are presumed.

${ }^{\#}$ Evaluated at $x=L_{\mathrm{b}}$.

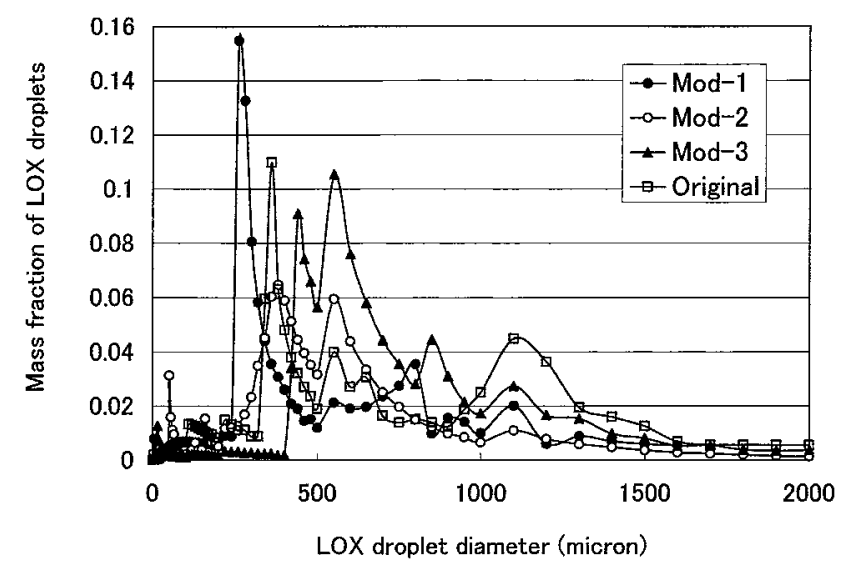

Fig. 12. Calculated histogram of LOX droplet's size distribution at $x=L_{\mathrm{b}}$ for optimization of injector.

\subsection{Optimization of design to improve combustion sta- bility}

As mentioned in the above discussion, CASE-D showed marginal stability of combustion. Therefore, redesign of the injection element for this case is attempted to improve combustion stability and performance. Here, the dimensions of the LOX injection orifice are considered as the main parameter for the redesign, and the temperature of injecting hydrogen is increased from 57 to $120 \mathrm{~K}$ to obtain better combustion performance. In Table 4 and Fig. 11, the adopted dimensions and the results of calculation are shown respectively. As a result, significant improvement in combustion stability is attained in the case of Mod-1-Mod-3. This is mainly because the atomization time delay is shortened by 
increasing the injection velocity of LOX. As for the $C^{*}$ efficiency, the case of Mod-1 and Mod-2 show better performance. This is because that both cases have a better histogram of the LOX droplet's size distribution, as shown in Fig. 12, in spite of having almost the same $D_{32}$ among these cases. Incidentally, the present model is able to provide optimization of the design to attain stable and higher combustion performance.

\section{Conclusion}

In this study, improved modeling of combustion processes was developed to evaluate combustion performance and combustion stability. The model was constructed by adopting the detailed mechanism of atomization characteristics of the coaxial injector of the liquid/gas phase, including the local rate of atomization and the size distribution of formed spray, as well by adopting the burning rate constant of LOX spray with gaseous hydrogen, which was obtained in the author's previous experiment. By applying this proposed model, the $C^{*}$ efficiencies for the LE-5 and its derivative engines were evaluated. The results showed reasonable agreement with the experimental data for the LE-5 (CASE-A) and its candidate engine (CASE-B). The combustion time delays, which were calculated by the present calculation model, were applied to Szuch's analytical model to evaluate combustion stability. As a result, it was shown that, in the case of the LE-5's derivative engine with a candidate injector, CASE-D, combustion was marginally stable. The present model also showed the usefulness of optimizing the design of that injector to improve its combustion stability and combustion performance. These facts show the predominance of the originality of the atomization mechanism of
LOX jet and the validity of the combustion model of LOX spray with gaseous hydrogen. In conclusion, it can be said that the present model provides a method for evaluating the design criteria of a LOX/hydrogen engine with a coaxial injector.

\section{References}

1) Yatsuyanagi, N.: An Empirical and Analytical Study of Spray Flow Fields Formed by Liquid-Gas Coaxial Injector Element. Part 3, Application for the Combustion Performance Prediction Model of LOX/ Hydrogen Rocket Combustor, Technical Report of the National Aerospace Laboratory, NAL TR-769, 1983 (in Japanese).

2) Priem, R.J. and Heidmann, M.F.: Propellant Vaporization as a Design Criterion for Rocket Engine Combustion Chambers, NASA TR-67, 1960.

3) Combs, L.P. and Schuman, M.D.: Steady State Rocket Combustion of Gaseous Hydrogen and Liquid Oxygen, Research Report RR64-29, Rocket-dyne, 1965.

4) Yatsuyanagi, N.: Study on Breakup Length of Liquid Jet by LiquidGas Coaxial Injector, Evaluation of Atomization Characteristics of Rocket Engine Injector, Trans. Jpn. Soc. Aeronaut. Space Sci., 49, 165 (2006), pp. 162-168.

5) Yatsuyanagi, N., Sakamoto, H. and Sato, K.: Experimental Study on Vaporization Rate of LOX-Spray with Methane and Hydrogen in Rocket Combustor, Proceedings of the 19th International Symposium on Space and Science, ISTS 94-a-21, 1994.

6) Yatsuyanagi, N.: An Empirical and Analytical Study of Spray Flow Fields Formed by Liquid-Gas Coaxial Injector Element (Part 2), Technical Report of the National Aerospace Laboratory, NAL TR-745, 1982 (in Japanese).

7) Yatsuyanagi, N., Sakamoto, H. and Sato, K.: Empirical Calculation Model of the Atomization of a Liquid Sheet and a Round Liquid Jet in a Gaseous Flow Field, Atomization and Spray, 8 (1998), pp. 267289.

8) Szuch, J. R.: Digital Computer Program for Analysis of Chugging Instability, NASA TN D-7026, 1970.

9) Penner, S.S. and Summerfield, Martin: Liquid Propellant Rockets, Princeton University Press, Princeton, N. J., 1960, p. 46. 